\title{
TRATAMENTO FARMACOLÓGICO DA HIPERALGESIA EXPERIMENTALMENTE INDUZIDA PELO NÚCLEO PULPOSO
}

\author{
PHARMACOLOGIC TREATMENT OF HYPERALGESIA \\ EXPERIMENTALLY INDUCED BY NUCLEUS PULPOSUS
}

André Luiz de Souza Grava', Luiz Fernando Ferrari' ${ }^{2}$, Carlos Amílcar Parada ${ }^{3}$, Helton Luiz Aparecido Defino ${ }^{4}$

\section{RESUMO}

Objetivo: Avaliar o efeito de drogas anti-inflamatórias (dexametasona, indometacina, atenolol, indometacina e atenolol) e analgésica (morfina) sobre a hiperalgesia experimentalmente induzida pelo núcleo pulposo em contato com o gânglio da raiz dorsal de L5. Métodos: Trinta ratos Wistar machos com peso de 220 a $250 \mathrm{~g}$ foram utilizados no estudo. A indução da hiperalgesia foi realizada por meio do contato de fragmento de núcleo pulposo retirado da região sacrococcígea e colocado sobre o gânglio da raiz dorsal de L5. Os 30 animais foram divididos em grupos experimentais de acordo com a droga utilizada. As drogas foram administradas durante duas semanas a partir da realização do procedimento cirúrgico para a indução da hiperalgesia. A hiperalgesia mecânica e térmica foram avaliadas por meio do teste da pressão constante da pata, von Frey eletrônico e Hargraves por um período de sete semanas. Resultados: A maior redução da hiperalgesia foi observada no grupo de animais tratados pela morfina, seguido pela dexametasona, indometacina e atenolol. A redução da hiperalgesia foi observada após a interrupção da administração das drogas, com exceção do grupo de animais tratados com morfina, nos quais ocorreu aumento da hiperalgesia após a interrupção do tratamento. Conclusões: A hiperalgesia induzida pelo contato do núcleo pulposo com o gânglio da raiz dorsal pode ser reduzida com a administração de antiinflamatórios e analgésicos, tendo sido observado a maior redução da hiperalgesia com a administração da morfina e dexametasona.

Descritores - Coluna Vertebral; Disco Intervertebral; Dor Lombar; Hiperalgesia; Deslocamento do Disco Intervertebral; Ratos Wistar

\section{ABSTRACT:}

Objective: To evaluate the effect of antiinflammatory (dexamethasone, indomethacin, atenolol, indomethacin and atenolol) and analgesic drugs (morphine) on hyperalgesia experimentally induced by nucleus pulposus (NP) contact with the L5- dorsal root ganglion (DRG). Methods: Thirty male Wistar rats with weights ranging from 220 to $250 \mathrm{~g}$ were used in the study. The hyperalgesia was induced by contact of a fragment of NP removed from the sacrococcygeal region and placed on the dorsal root ganglion of L5. The 30 animals were divided into experimental groups according to the drug administered. The drugs were administered during the two weeks after the surgical procedure to induce hyperalgesia. Mechanical and thermal hyperalgesia was evaluated by the paw pressure test, von Frey electronic test, and the Hargraves test, for a period of seven weeks. Results: The greatest reduction of hyperalgesia was observed in animals treated by morphine and dexamethasone, followed by dexamethasone, indomethacin, and atenolol. The reduction of hyperalgesia was observed after drug administration ceased, except for animals treated with morphine, in which there was an increased hyperalgesia after cessation of treatment. Conclusion: Hyperalgesia induced by NP contact with L5-DRG can be reduced by administration of antiinflammatory and analgesic drugs, but there was a greater reduction observed with the administration of dexamethasone and indometacin

Keywords - Spine; Intervertebral Disk; Low Back Pain; Hyperalgesia; Intervertebral Disk Displacement; Ratos Wistar

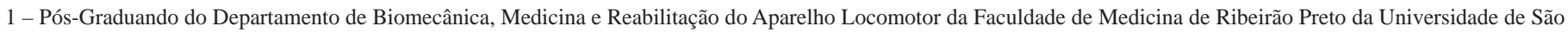
Paulo - USP - Ribeirão Preto (SP), Brasil.

2 - Pós-Graduando, Departamento de Farmacologia da Faculdade de Medicina de Ribeirão Preto da Universidade de São Paulo - USP - Ribeirão Preto (SP), Brasil.

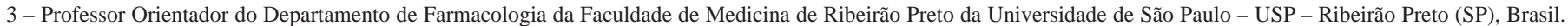

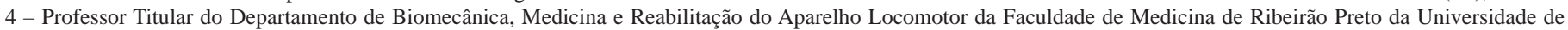
São Paulo - USP - Ribeirão Preto (SP), Brasil.

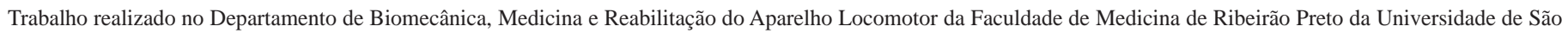
Paulo - USP - Ribeirão Preto (SP) - Brasil.

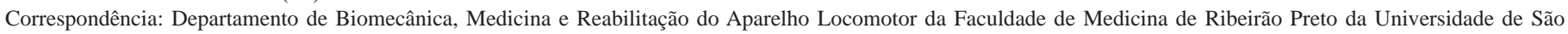
Paulo - USP. Av. Bandeirantes, 3.900, 11º andar - 14048-900 - Ribeirão Preto (SP). E-mail: hladefin@fmrp.usp.br

Trabalho recebido para publicação: 12/01/10, aceito para publicação: 09/03/10. 


\section{INTRODUÇÃO}

A fisiopatologia da hérnia de disco lombar está relacionada com a compressão mecânica da raiz nervosa e alterações induzidas pelo contato do núcleo pulposo com os elementos nervosos ${ }^{(1,2)}$. Estudos experimentais têm demonstrado que na ausência da compressão mecânica da raiz nervosa o contato do núcleo pulposo induz a alterações funcionais e estruturais da raiz nervosa ${ }^{(3,4)}$.

O mecanismo de indução do processo inflamatório pelo núcleo pulposo permanece desconhecido e a irritação química direta e a reação imunológica secundária dos tecidos adjacentes à presença do núcleo pulposo têm sido considerados como os prováveis mecanismos ${ }^{(5)}$.

A sensibilização das fibras nervosas sensitivas, dentre elas as nociceptivas, é fenômeno predominante e é o denominador comum da dor inflamatória que induz o aparecimento da hiperalgesia por meio de mecanismos periféricos ou medulares ${ }^{(6)}$. As prostaglandinas e as aminas simpaticomiméticas participam do processo inflamatório, e drogas anti-inflamatórias e analgésicas interferem nesse processo inflamatório ${ }^{(6)}$. O objetivo do estudo foi avaliar o efeito de diferentes drogas (dexametasona, indometacina, atenolol e morfina) sobre a hiperalgesia induzida pelo núcleo pulposo sobre o gânglio da raiz nervosa na ausência de compressão mecânica.

\section{MATERIAL E MÉTODOS}

O protocolo do estudo foi aprovado pelo Comitê de Ética em Experimentação Animal da Faculdade de Medicina de Ribeirão Preto e realizado de acordo com as normas internacionais de ética para a utilização de animais de laboratório. Foram utilizados 30 ratos Wistar machos, pesando entre $220 \mathrm{~g}$ e $250 \mathrm{~g}$. Antes da realização dos experimentos, os animais permaneceram dois dias no biotério local para aclimatação, em gaiolas de 40 x 60 x 20cm, com o máximo de 06 ratos por gaiola, sob condições controladas de temperatura (22 a $25^{\circ} \mathrm{C}$ ) e ciclo claro/escuro (12 x 12h), com livre acesso à comida e água, antes de serem submetidos aos ensaios. O modelo experimental utilizado no estudo consistia de procedimento cirúrgico para a remoção do núcleo pulposo da região sacrococcígea e sua colocação sobre o gânglio da raiz dorsal (GRD) de L5 ${ }^{(7)}$. Após o procedimento cirúrgico os animais eram divididos em grupos experimentais com cinco animais em cada grupo. Os grupos foram determinados de acordo com a droga administrada (dexametasona, indometacina, atenolol, indometacina + atenolol, morfina e solução salina).

\section{Procedimento cirúrgico}

Protocolo cirúrgico - Os animais foram anestesiados com injeção intraperitoneal de ketamina $10 \%$ $(0,1 \mathrm{ml} / 100 \mathrm{~g})$, xilazina $2 \%(0,07 \mathrm{ml} / 100 \mathrm{~g})$ e fentanil $5 \%$ (0,001ml/100g).

O núcleo pulposo (NP) foi removido da região sacroccígea (base da cauda do rato) por meio de incisão mediana na transição da quarta vértebra sacral e primeira vértebra coccígea. O disco intervertebral foi bilateralmente exposto e o NP removido por meio de incisão transversal do ânulo fibroso. O NP foi coletado (Figura 1), pesado em balança de precisão (peso médio de 4 to $5 \mu \mathrm{g}$ ) e colocado sobre o GRD-L5, que foi exposto por meio de laminectomia parcial. A cirurgia para a exposição do GRD-L5 e colocação do fragmento do NP foi realizada por meio de lente de aumento de 10x. Após o procedimento, a ferida cirúrgica foi fechada em único plano envolvendo a fascia muscular e a pele.
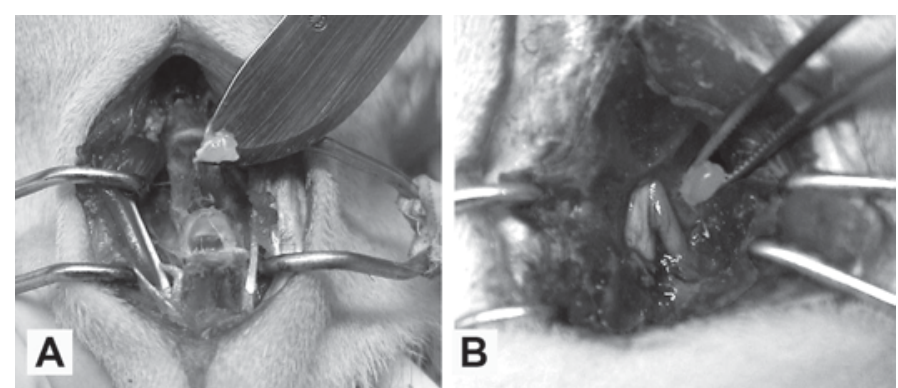

Figura 1 - Exposição do disco intervertebral da região sacrococcígea (A) e deposição do fragmento do NP sobre o gânglio da raiz dorsal de $L 5(B)$.

\section{Grupos experimentais}

Os animais foram divididos em seis grupos experimentais com cinco animais em cada grupo, de acordo com a droga administrada. As drogas foram administradas por um período de 15 dias após a realização do procedimento cirúrgico para a colocação do NP em contato com o GRD-L5. Com exceção da morfina, que era administrada 30 minutos antes da realização dos testes para avaliação da hiperalgesia, as demais drogas foram diariamente administradas durante duas semanas após a realização da cirurgia. A morfina foi administrada no terceiro dia, primeira e segunda semanas após a cirurgia.

Grupo I - dexametasona - 0,5mg/kg - subcutânea.

Grupo II - indometacina - 2mg/kg - subcutânea.

Grupo III - atenolol - 1mg/kg - intraperitoneal.

Grupo IV - indometacina (2mg/kg - subcutânea) + atenolol (1mg/kg - intraperitoneal)

Grupo V - morfina - 4mg/kg - intraperitoneal.

Grupo VI - solução salina - 3ml - intraperitoneal. 


\section{Avaliação da hiperalgesia}

A avaliação da hiperalgesia foi realizada para a determinação do limiar de tolerância para estímulos mecânicos (teste da pressão constante da pata e teste de von Frey eletrônico) ${ }^{(8,9)}$ e térmicos (teste de Hargreaves) $)^{(10)}$ antes do procedimento cirúrgico e após a administração das drogas. Os testes foram realizados no terceiro dia de pós operatório e semanalmente até a sétima semana após a realização do procedimento cirúrgico. Os testes foram realizados por examinadorindependente e sem o conhecimento do grupo experimental dos animais. Os teste para a avaliação da hiperalgesia foram realizados durante a administração das drogas (duas semanas) e durante cinco semanas adicionais após a sua interrupção tendo sido realizado por um período total de sete semanas.

Teste da pressão constante da pata - a latência da resposta nociceptiva foi realizada por meio do teste descrito por Randall e Selitto ${ }^{(8)}$. Nesse método uma pressão constante de $20 \mathrm{mmHg}$ (medida com o uso de um esfigmomanômetro) é aplicada por meio de um pistão de uma seringa movida pela compressão do ar, em uma área de $15 \mathrm{~mm}^{2}$, na superfície dorsal das patas traseiras dos ratos. A pressão é descontinuada quando o animal apresenta uma "reação típica", interpretada como nociceptiva. Essa "reação típica” é sinalizada por uma breve apneia, com concomitante retração das patas dianteiras. Para cada animal é determinado o tempo de latência desde a aplicação da pressão e o aparecimento da reação típica. A intensidade da hiperalgesia é quantificada por meio da redução da diferença do tempo, calculado pela subtração do tempo de latência em segundos, antes e após o tratamento empregado.

Teste de pressão crescente na pata de ratos (Von Frey Eletrônico) - A hiperalgesia mecânica foi avaliada por meio do teste de pressão crescente na pata de ratos, denominado de teste von Frey eletrônico (tenocicepção) ${ }^{(9)}$. O método consiste no uso de um anestesiômetro eletrônico (modelo 1601C, Life Science Instruments ${ }^{\circledR}$ ), o qual possui um transdutor de força conectado a um contador digital de força expressa em gramas (g). A precisão do aparelho é de 0,1g e o mesmo é calibrado para registrar força máxima de $150 \mathrm{~g}$, mantendo a precisão de $0,1 \mathrm{~g}$ até a força de $80 \mathrm{~g}$. O contato do transdutor de força com a pata é realizado por meio de uma ponteira descartável de polipropileno com $0,5 \mathrm{~mm}$ de diâmetro adaptada ao transdutor. Os animais são colocados em caixas de acrílico, medindo $12 \times 20 \times 17 \mathrm{~cm}$, cujo assoalho consiste de uma rede de malha igual a $5 \mathrm{~mm}^{2}$, constituída de arame não maleável de $1 \mathrm{~mm}$ de espessura, durante 15 minutos antes do experimento, para adaptação ao ambiente. Espelhos posicionados $25 \mathrm{~cm}$ abaixo das caixas de experimentação facilitam a visualização das plantas das patas dos animais. $\mathrm{O}$ experimentador aplica, por entre as malhas da rede, força linearmente crescente no centro da planta da pata do rato até que o animal produza uma resposta de retirada e "sacudida" da pata estimulada. Os estímulos são repetidos até seis vezes, em geral até que o animal apresente três medidas similares com uma nítida resposta de "sacudida" após a retirada da pata.

Teste de hiperalgesia térmica de Hargreaves (Plantar Test) - A avaliação da hiperalgesia térmica foi realizada por meio do teste plantar de Hargreaves et $a l^{(10)}$. Esse teste consiste no aquecimento, por meio de fonte de luz infravermelha dirigida, da região plantar da pata traseira do rato, até que o animal apresente o comportamento da retirada da pata ou o aquecimento da pata atinja o limite de tempo predeterminado (tempo de corte de 12 segundos).

Os animais são colocados em compartimentos de acrílico individuais e posicionados sobre uma superfície de vidro especial, que permite a passagem de forma homogênea da luz e do calor, durante cinco minutos, para adaptação ao ambiente. Após esse período, uma fonte de luz infravermelha, colocada sob cada uma das patas traseiras do animal, é acionada juntamente com um cronômetro eletrônico, até que o animal retire a pata, quando, então, a fonte de luz e o relógio param automaticamente.

São realizadas três medidas com intervalo de tempo de cinco a $10 \mathrm{~min}$. As medidas de latência de retirada da pata são então expressas como avaliações percentuais relativas às patas-controle (contralaterais) em cada tempo de observação. Na situação em que o animal não apresenta reação, é estipulado um tempo limite máximo de corte do experimento de 12 segundos para a preservação da sua integridade física.

Estudo estatístico - Os resultados serão apresentados como a média dos valores de cada grupo experimental de cinco animais e o seu desvio padrão. A avaliação estatística foi realizada por meio do teste one-way ANOVA seguido do teste de Bonferroni, tendo sido estabelecido o nível de significância de $\mathrm{p}<0,05$.

\section{RESULTADOS}

A administração das drogas anti-inflamatórias evidenciou a sua capacidade de redução da hiperalgesia mecânica e térmica induzida pelo contato do núcleo 
pulposo com o gânglio da raiz dorsal (Figuras 2, 3, 4, 5 e 6). A dexametasona, dentre as drogas utilizadas no

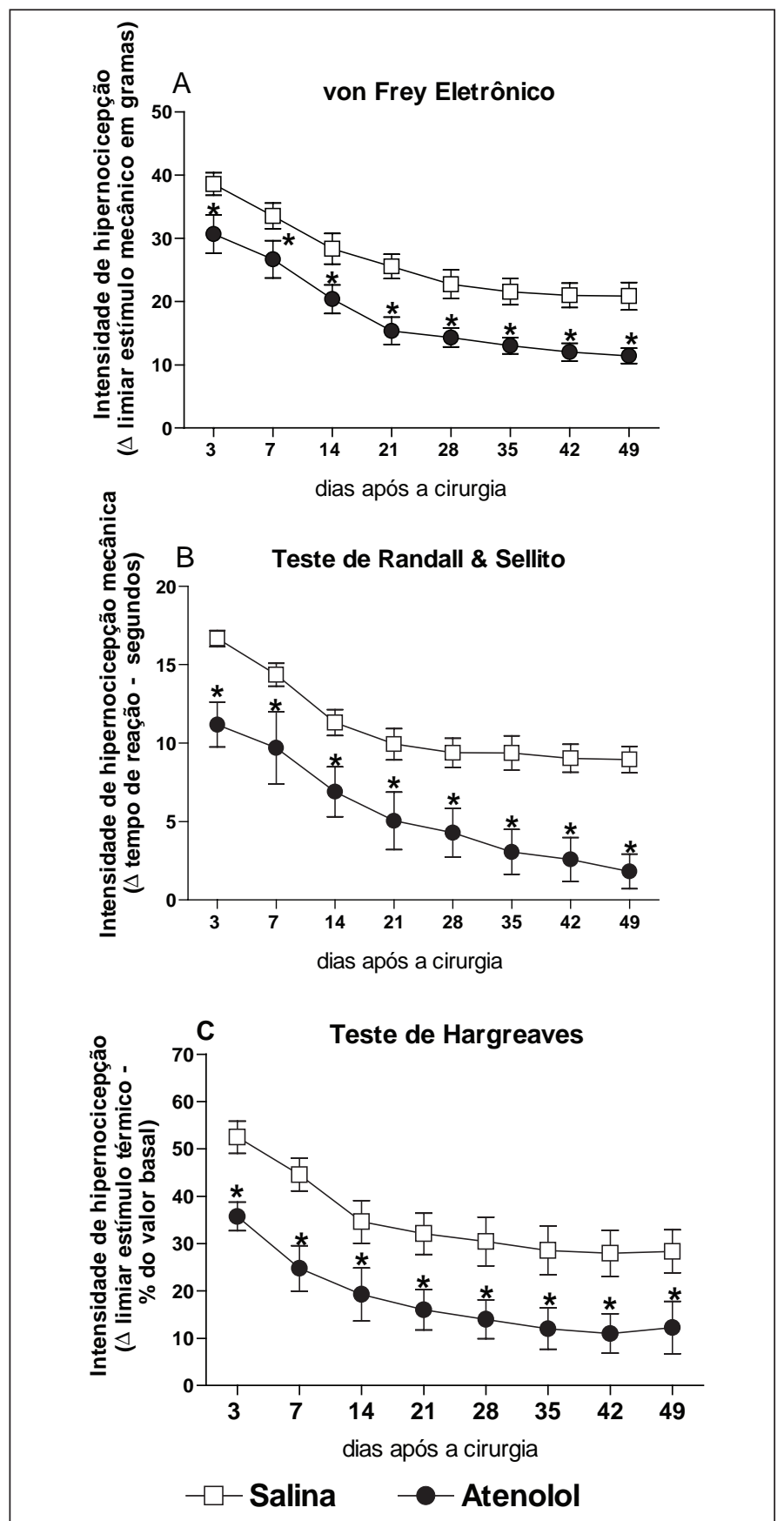

Figura 2 - Intensidade de hiperalgesia em animais submetidos à cirurgia de indução de hérnia de disco lombar tratados no período pós-operatório com atenolol. Após a cirurgia, os animais foram tratados com atenolol $(1,0 \mathrm{mg} / \mathrm{kg})$ durante os primeiros sete dias e foram acompanhados durante 49 dias, com medidas semanais. A intensidade de hiperalgesia foi avaliada em três diferentes testes comportamentais: teste de pressão crescente na pata de ratos (von Frey eletrônico) (A), teste de pressão constante na pata de ratos (Randall e Sellito modificado) (B) e teste térmico de Hargreaves (C). As medidas representam a média \pm EPM de seis animais por grupo. ${ }^{*} p<0,05$ quando comparado ao grupo controle tratado com solução salina (MANOVA, seguida de teste $t$ de Student com desigualdade de Bonferroni).
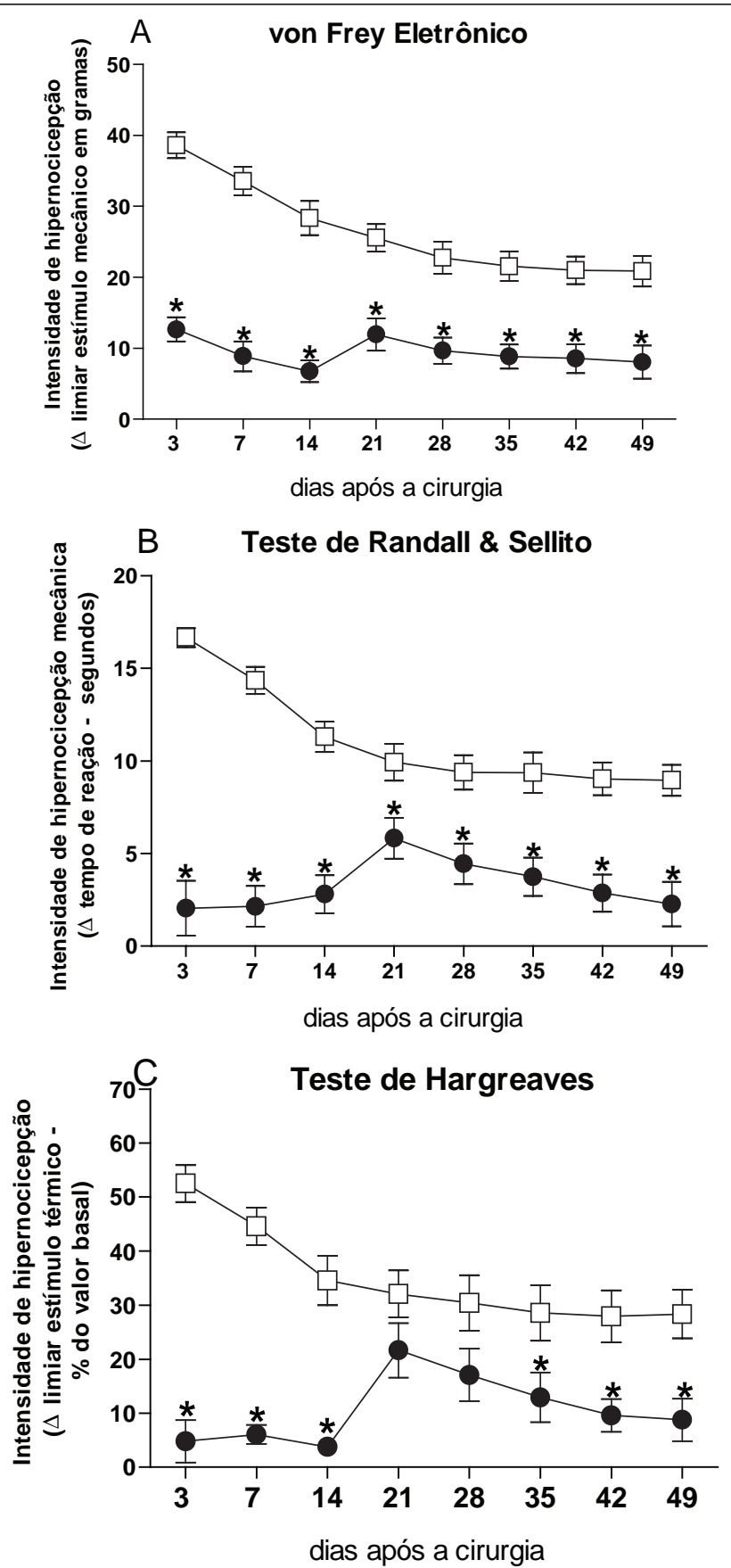

Salina

Dexametasona

Figura 3 - Intensidade de hiperalgesia em animais submetidos à cirurgia de indução de hérnia de disco lombar tratados no período pós-operatório com Dexametasona. Após a cirurgia, os animais foram tratados com dexametasona $(0,5 \mathrm{mg} / \mathrm{kg})$ durante os primeiros sete dias e foram acompanhados durante 49 dias, com medidas semanais. A intensidade de hiperalgesia foi avaliada em três diferentes testes comportamentais: teste de pressão crescente na pata de ratos (von Frey eletrônico) (A), teste de pressão constante na pata de ratos (Randall e Sellito modificado) $(B)$ e teste térmico de Hargreaves $(\mathrm{C})$. As medidas representam a média \pm EPM de seis animais por grupo. ${ }^{*} \mathrm{p}<0,05$ quando comparado ao grupo controle tratado com solução salina (MANOVA, seguida de teste $t$ de Student com desigualdade de Bonferroni). 


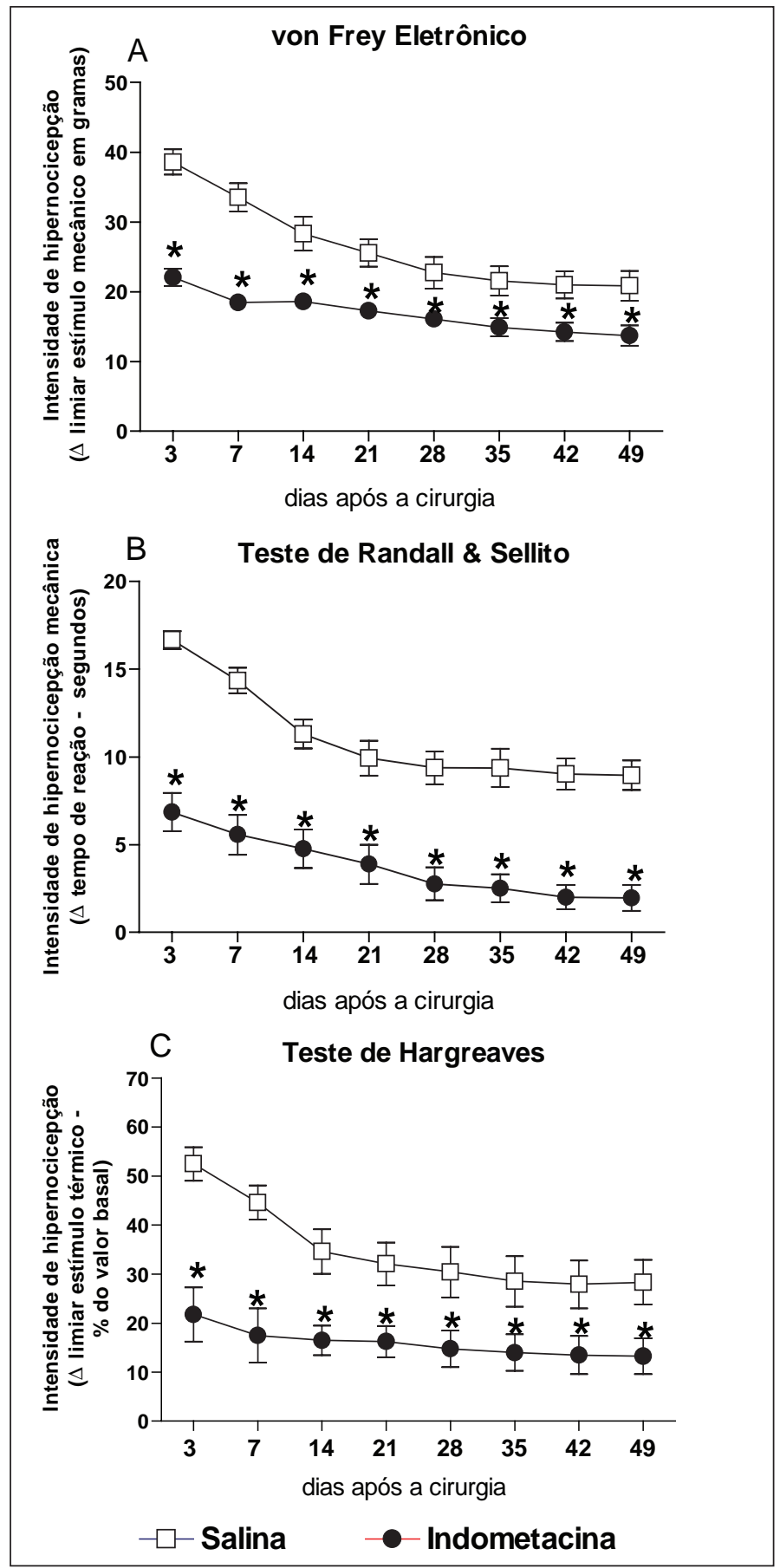

Figura 4 - Intensidade de hiperalgesia em animais submetidos à cirurgia de indução de hérnia de disco lombar tratados no período pós operatório com indometacina. Após a cirurgia, os animais foram tratados com indometacina $(2,0 \mathrm{mg} / \mathrm{kg})$ durante os primeiros sete dias e foram acompanhados durante 49 dias, com medidas semanais. A intensidade de hiperalgesia foi avaliada em três diferentes testes comportamentais: teste de pressão crescente na pata de ratos (von Frey eletrônico) (A), teste de pressão constante na pata de ratos (Randall e Sellito modificado) (B) e teste térmico de Hargreaves (C). As medidas representam a média \pm EPM de seis animais por grupo. * $p<$ 0,05 quando comparado ao grupo controle tratado com solução salina (MANOVA, seguida de teste $t$ de Student com desigualdade de Bonferroni).

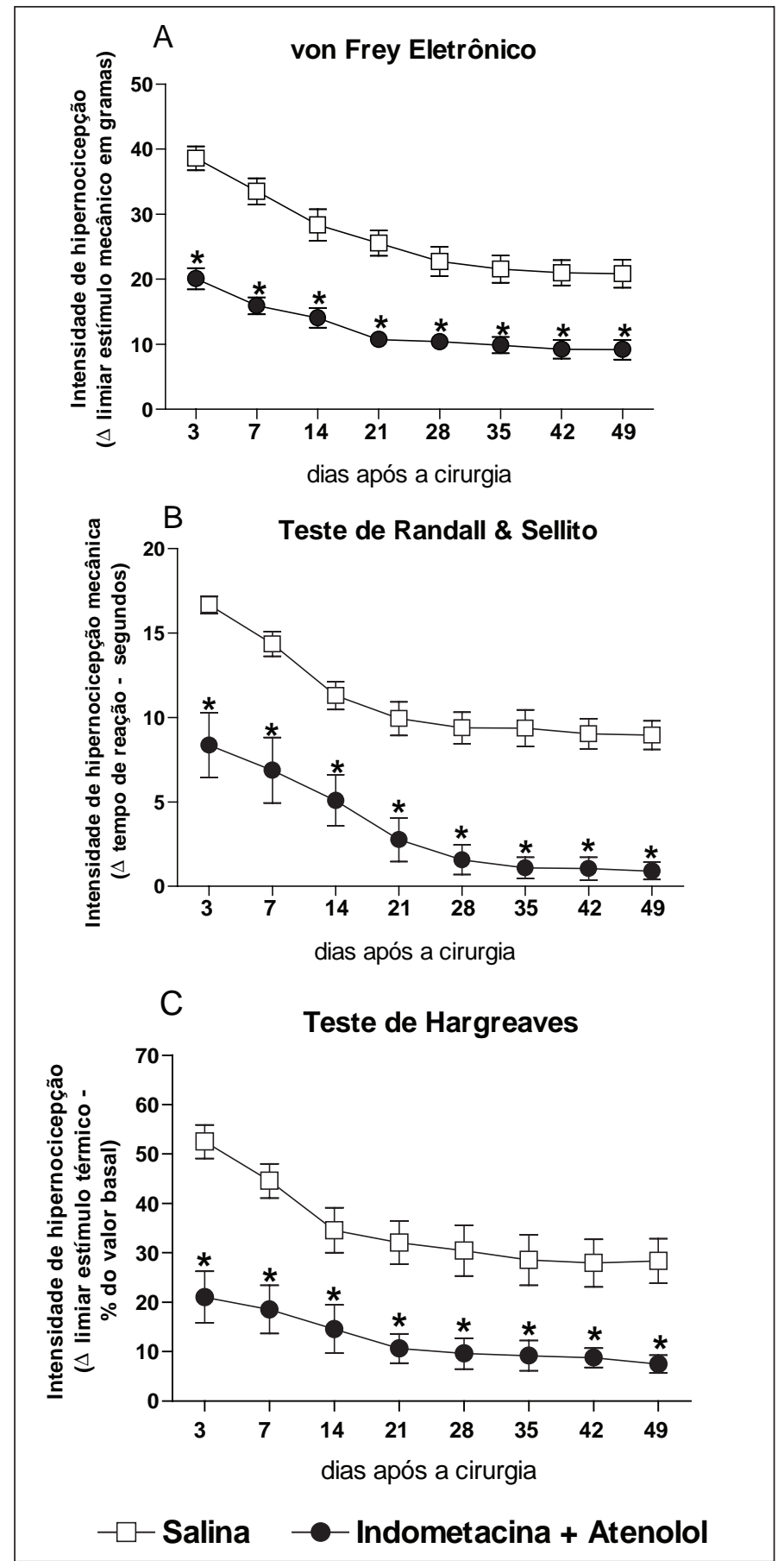

Figura 5 - Intensidade de hiperalgesia em animais submetidos à cirurgia de indução de hérnia de disco lombar tratados com indometacina e atenolol. Após a cirurgia, os animais foram tratados com indometacina $(2,0 \mathrm{mg} / \mathrm{kg})$ e atenolol $(1,0 \mathrm{mg} /$ $\mathrm{kg}$ ) simultaneamente, durante os primeiros sete dias e foram acompanhados durante 49 dias, com medidas semanais. A intensidade de hiperalgesia foi avaliada em três diferentes testes comportamentais: teste de pressão crescente na pata de ratos (von Frey eletrônico) (A), teste de pressão constante na pata de ratos (Randall e Sellito modificado) (B) e teste térmico de Hargreaves $(\mathrm{C})$. As medidas representam a média \pm EPM de seis animais por grupo. * $p<0,05$ quando comparado ao grupo controle tratado com solução salina (MANOVA, seguida de teste $t$ de Student com desigualdade de Bonferroni). 

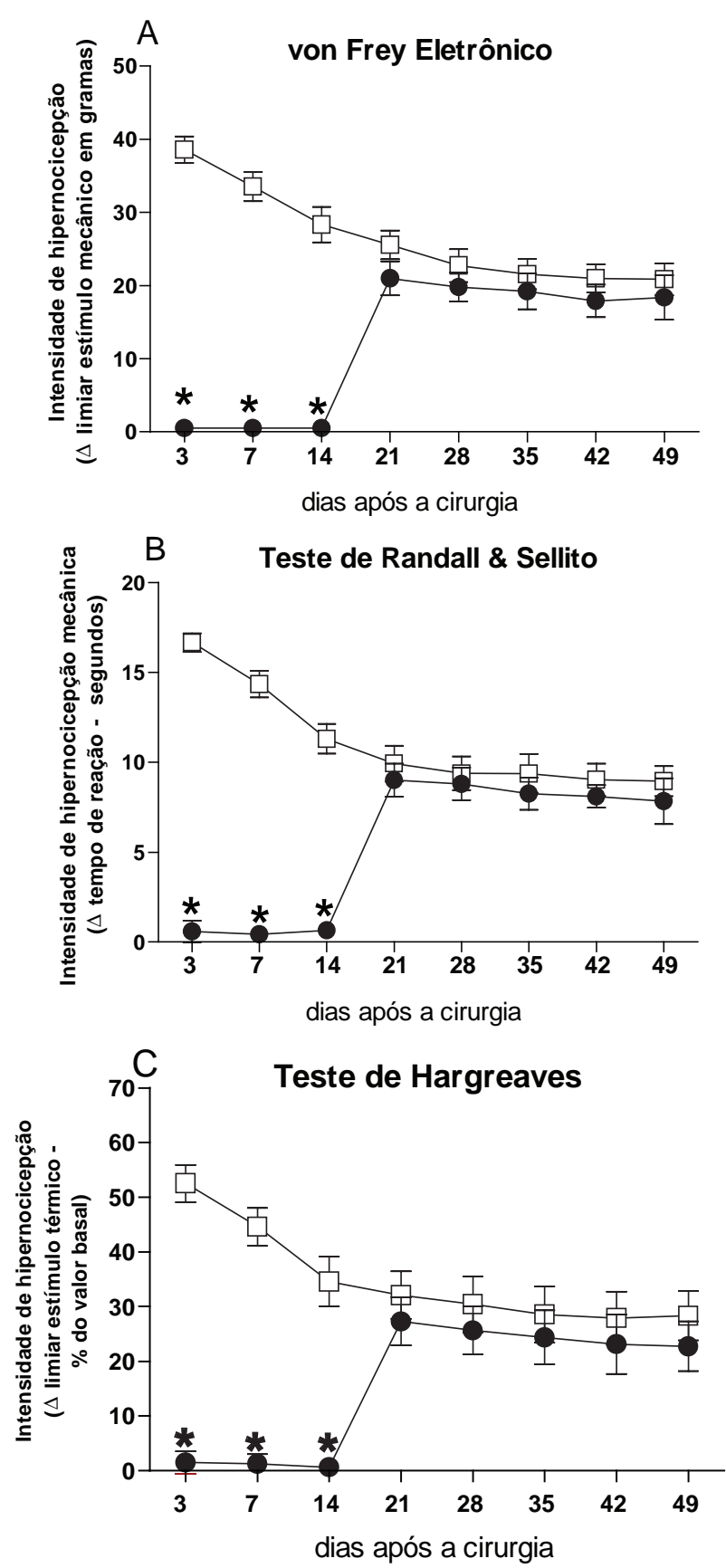

$-\square$ Salina $\quad \longrightarrow$ Morfina

Figura 6 - Intensidade de hiperalgesia em animais submetidos à cirurgia de indução de hérnia de disco lombar tratados com Morfina. Após a cirurgia, os animais foram tratados com morfina $(4 \mathrm{mg} / \mathrm{kg})$, durante os primeiros sete dias e foram acompanhados durante 49 dias, com medidas semanais. A intensidade de hiperalgesia foi avaliada em três diferentes testes comportamentais: teste de pressão crescente na pata de ratos (von Frey eletrônico) $(A)$, teste de pressão constante na pata de ratos (Randall e Sellito modificado) (B) e teste térmico de Hargreaves (C). As medidas representam a média \pm EPM de seis animais por grupo. * $p<0,05$ quando comparado ao grupo controle tratado com solução salina (MANOVA, seguida de teste $t$ de Student com desigualdade de Bonferroni). estudo, foi a que apresentou a maior redução da hiperalgesia, tendo sido observado aumento após o término da sua administração. O atenolol e a indometacina reduziram a intensidade hiperalgesia, mas de menor intensidade em relação à dexametasona. A associação do atenolol e indometacina apresentou redução mais intensa da hiperalgesia que a administração isoladas dessas drogas.

A administração da morfina provocou a maior redução da intensidade da hiperalgesia nos três testes realizados. Após o término da sua administração os valores do grupo tratado não apresentaram diferença em relação ao grupo controle. Ao contrário do observado com a administração das drogas anti-inflamatórias, a redução da hiperalgesia provocada pela morfina não se manteve após a interrupção da sua administração.

\section{DISCUSSÃO}

O processo inflamatório induzido pelo contato do NP com as estruturas nervosas tem sido amplamente relatado ${ }^{(2)}$. A sensibilização dos receptores é o denominador comum do processo inflamatório envolvido com o mecanismo da dor inflamatória. A regulação funcional dos receptores da dor conduz ao estado de hiperalgesia ou alodinia, e existe dois grupos de mediadores que estão envolvidos nesse processo e que atuam diretamente na sensibilização dos nociceptores: os eicosanoides e as aminas simpatomiméticas ${ }^{(6)}$. A capacidade desses mediadores para a sensibilização dos receptores da dor tem sido demonstrada em animais e humanos ${ }^{(6)}$.

Os mediadores inflamatórios provêm basicamente de três fontes: sangue, células residentes e do próprio nociceptor. Essas substâncias podem ativar diretamente (bradicinina, serotonina, histamina em altas doses), sensibilizar indiretamente (bradicinina, histamina, substância P, TNF- $\alpha$, leucotrienos e citocinas) ou ainda, sensibilizar diretamente (prostaglandinas, serotonina, noradrenalina) os nociceptores ${ }^{(11-15)}$.

Em modelo experimental desenvolvido para essa finalidade, observamos os efeitos do contato do NP sobre o GRD, que definiram o modelo utilizado neste estudo ${ }^{(7)}$. O estudo foi delineado considerando a indução da hiperalgesia que ocorre devido ao processo inflamatório decorrente do contato dos elementos do NP com o RDG.

Este estudo foi realizado com o objetivo de fornecer conhecimentos sobre o mecanismo da hiperalgesia induzida pelo NP, utilizando drogas cujo sítio e mecanismos de ação são conhecidos. 
O tratamento com a indometacina, anti-inflamatório não esteroidal, foi capaz de reduzir a intensidade da hiperalgesia em cerca de 50\% em relação ao grupo controle, na avaliação inicial realizada no terceiro dia. O atenolol (inibidor das aminas simpatomiméticas) mostrou menor redução da intensidade da dor aguda. A associação da indometacina com o atenolol mostrou semelhança com a redução induzida pela indometacina, sugerindo que nessa fase do processo a participação das prostaglandinas deva ser maior que das aminas simpatomiméticas na produção da hiperalgesia. Após a instalação do quadro crônico de hiperalgesia (14 dias) os resultados da administração do atenolol passaram a ser semelhantes aos da administração de indometacina, sugerindo a participação das aminas simpatomiméticas possa ser mais relevante nessa fase.

A hipersensibilidade neuronal produzida durante o processo inflamatório possui dois componentes distintos, sendo um deles dependente da ativação da enzima ciclooxigenase $(\mathrm{COX}) 1$ e 2, conduzindo à produção de eicosanoides (o mais importante sendo a prostaglandina), e o outro dependente da liberação de aminas simpatomiméticas, como a noradrenalina ${ }^{(13,16)}$. As drogas anti-inflamatórias não esteroidais (AINEs) poderiam apresentar certa limitação da sua eficácia, considerando o seu mecanismo de ação de inibição das $\operatorname{COXs}^{(17,18)}$. Os resultados sugerem que na fase aguda o componente prostaglandínico possui maior relevância na indução da hiperalgesia. No entanto, os resultados obtidos com a administração do inibidor de receptores beta-adrenérgicos (atenolol) indica que em algum momento possa ocorrer também a participação desse componente de modo mais pronunciado que as prostaglandinas, principalmente se considerarmos a possibilidade do desenvolvimento de características de dor neuropática, pois as aminas simpatomiméticas podem estar envolvidas nessa transição. Estudos experimentais sugerem esse efeito, mesmo que o componente inflamatório ainda esteja ativo ${ }^{(19,20)}$. Poderia ocorrer a mudança do estado inflamatório, tendo como protagonistas os eicosanoides produzidos pela ativação da COX, para um estado neuropático, com a atuação das aminas simpatomiméticas produzidas pelo segundo braço da cascata de citocinas, explicando desse modo a observação dos efeitos semelhantes do atenolol ao da indometacina após a cronificação do quadro depois de 14 dias.

A dexametasona produziu a maior redução da hiperalgesia na fase aguda, semelhante ao que tem sido observado com a sua utilização clínica. Apesar do mecanismo de ação dos corticosteroides serem extremamente diversificados, com estudos mostrando novas formas de ação para indução da analgesia e redução do processo inflamatório ${ }^{(21)}$, a inibição da síntese das prostaglandinas e das aminas simpatomiméticas poder ter sido a responsável pelo seu intenso efeito sobre a redução da hiperalgesia. Outro modo adicional de ação seria a sua ação sobre o TNF- $\alpha$, cuja participação tem sido demonstrada na indução da hiperalgesia que ocorre na hérnia de $\operatorname{disco}^{(22)}$.

A administração da morfina reduziu de modo considerável a hiperalgesia mecânica e térmica, e após a interrupção da sua administração ocorreu a recidiva da hiperalgesia, com a sua intensidade apresentando valores semelhantes ao grupo tratado com solução salina. Esse efeito temporário do opioide, observado apenas durante a sua administração poderia ser explicado pelas recentes observações de que apenas a fibras neuronais relacionadas com a dor e a nocicepção (fibras do tipo C) apresentariam receptores para a morfina ${ }^{(6)}$. A participação real e exclusiva das fibras nociceptoras $\mathrm{C}$ merece estudos futuros, pois se realmente apenas as fibras $\mathrm{C}$ possuírem receptores para a morfina, pelo menos na fase aguda pode ser que apenas esse tipo de fibra nervosa seja a responsável pela sensibilidade mecânica e térmica. No entanto, tem sido sugerida a ocorrência de alteração de fibras do tipo A-delta, que mediante o processo de injuria passariam a inervar camadas da medula espinhal cujos neurônios conduzem estímulos de diversos tipos como o tato ${ }^{(23,24)}$. Esses neurônios são conhecidos como WDR (wide dinamic range), seriam ativados pelas fibras A-delta sensibilizadas e contribuiriam para a observação da alodinia. Se essas fibras não forem afetadas pela morfina, e sendo essas participantes de processos neuropáticos, poderia ser que a continuidade do tratamento com a morfina não tivesse efeito tão pronunciado em períodos mais tardios do tratamento, provavelmente pela instalação de um processo neuropático após o término da fase aguda.

A administração de diferentes classes de drogas antiinflamatórias e analgésica reduziu a intensidade da hiperalgesia induzida pelo contato do NP com o GRD-L5. A ação das drogas ocorreria pela sua atuação sobre a cascata do processo inflamatório desencadeado pelo NP, e as diferentes respostas evidenciam a intensidade da participação dos eicosanoides e aminas simpatomiméticas nesse processo.

\section{CONCLUSÕES}

A administração de diferentes tipos de drogas antiinflamatórias reduziu a intensidade da hiperalgesia experimental induzida pelo contato do NP com o GRD-L5, e a dexametasona apresentou o maior efeito. 


\section{REFERÊNCIAS}

1. Jonsson B, Stromqvist B. Clinical appearance of contained and noncontained lumbar disc herniation. J Spinal Disord. 1996;9(1):32-8.

2. Olmarker K, Storkson R, Berge OG. Pathogenesis of sciatic pain: a study of spontaneous behavior in rats exposed to experimental disc herniation. Spine. 2002;27(12):1312-7.

3. Olmarker K, Rydevik B, Nordborg C. Autologous nucleus pulposus induces neurophysiologic and histologic changes in porcine cauda equina nerve roots. Spine (Phila Pa 1976). 1993;18(11):1425-32.

4. Otani K, Arai I, Mao GP, Konno S, Olmarker K, Kikuchi S. Experimental disc herniation: evaluation of the natural course. Spine (Phila Pa 1976). 1997;22(24):2894-9.

5. Kawakami M, Tamaki T, Matsumoto T, Kuribayashi K, Takenaka T, Shinozaki M. Role of leukocytes in radicular pain secondary to herniated nucleus pulposus. Clin Orthop Relat Res. 2000;(376):268-77.

6. Sachs D, Cunha FQ, Poole S, Ferreira SH. Tumour necrosis factor-alpha, interleukin-1beta and interleukin-8 induce persistent mechanical nociceptor hypersensitivity. Pain. 2002;96(1-2):89-97.

7. Grava AL, Ferrari LF, Parada CA, Defino HL. Experimental model to study intervertebral disc herniation. Rev Bras Ortop. 2008;43(4):116-25.

8. Randall LO, Selitto JJ. A method for measurement of analgesic activity on inflamed tissue. Arch Int Pharmacodyn Ther. 1957;111(4):409-19.

9. Vivancos GG, Verri WA Jr, Cunha TM, Schivo IR, Parada CA, Cunha FQ, et al. An electronic pressure-meter nociception paw test for rats. Braz J Med Biol Res. 2004;37(3):391-9.

10. Hargreaves K, Dubner R, Brown F, Flores C, Joris J. A new and sensitive method for measuring thermal nociception in cutaneous hyperalgesia. Pain. 1988;32(1):77-88

11. Birrell GJ, McQueen DS. The effects of capsaicin, bradykinin, PGE2 and cicaprost on the discharge of articular sensory receptors in vitro. Brain Res. 1993;611(1):103-7.

12. Dray A, Bettaney J, Forster P, Perkins MN. Activation of a bradykinin receptor in peripheral nerve and spinal cord in the neonatal rat in vitro. $\mathrm{Br} \mathrm{J}$ Pharmacol.
1988:95(4):1008-10.

13. Ferreira SH, Nakamura MI. Prostaglandin hyperalgesia, a cAMP/Ca2+ dependent process. Prostaglandins. 1979;18(2):179-90.

14. Millan MJ. The induction of pain: an integrative review. Prog Neurobiol. 1999;57(1):1-164.

15. Schepelmann K, Messlinger K, Schaible HG, Schmidt RF. Inflammatory mediators and nociception in the joint: excitation and sensitization of slowly conducting afferent fibers of cat's knee by prostaglandin 12. Neuroscience. 1992;50(1):237-47.

16. Gold MS, White DM, Ahlgren SC, Guo M, Levine JD. Catecholamine-induced mechanical sensitization of cutaneous nociceptors in the rat. Neurosci Lett. 1994;175(1-2):166-170.

17. Vane JR, Botting RM. Mechanism of action of antiinflammatory drugs. Int J Tissue React. 1998;20(1):3-15.

18. Vane JR, Botting RM. Mechanism of action of nonsteroidal anti-inflammatory drugs. Am J Med. 1998;104(3A):2S-8S.

19. Moon DE, Lee DH, Han HC, Xie J, Coggeshall RE, Chung JM. Adrenergic sensitivity of the sensory receptors modulating mechanical allodynia in a rat neuropathic pain model. Pain. 1999;80(3):589-95.

20. Nam TS, Yeon DS, Leem JW, Paik KS. Adrenergic sensitivity of uninjured C-fiber nociceptors in neuropathic rats. Yonsei Med J. 2000;41(2):252-7.

21. Barnes PJ. How corticosteroids control inflammation: Quintiles Prize Lecture 2005. Br J Pharmacol. 2006;148(3):245-54.

22. Igarashi T, Kikuchi S, Shubayev V, Myers RR. 2000 Volvo Award winner in basic science studies: exogenous tumor necrosis factor-alpha mimics nucleus pulposus-induced neuropathology. Molecular, histologic, and behavioral comparisons in rats. Spine (Phila Pa 1976). 2000;25(23):2975-80.

23. Farajidavar A, Gharibzadeh S, Towhidkhah F, Saeb S. A cybernetic view on wind-up. Med Hypotheses. 2006;67(2):304-6.

24. Hu J, Mata M, Hao S, Zhang G, Fink DJ. Central sprouting of uninjured small fiber afferents in the adult rat spinal cord following spinal nerve ligation. Eur $\mathrm{J}$ Neurosci. 2004;20(7):1705-12. 